\title{
Sudden shortening of the paced AV delay: is this normal pacemaker function?
}

\author{
Arnold Pinter $\cdot$ Paul Dorian
}

Published online: 14 January 2015

(C) The Author(s) 2014. This article is published with open access at Springerlink.com

A 74-year-old female patient underwent a Medtronic KDR601 dual chamber pacemaker implantation for syncope due to sinus node disease. The ventricular and atrial passive fixation pacemaker leads were positioned in the right ventricular apex and right atrial appendage, respectively. The sensing and capture threshold values were satisfactory in both chambers. The device was programmed to DDDR mode 60-130 ppm with both the upper tracking rate and maximum sensor rate at $130 \mathrm{ppm}$, paced AV delay at $200 \mathrm{~ms}$, and sensed AV delay at $180 \mathrm{~ms}$. Postoperative chest $\mathrm{X}$-ray showed normal pacemaker lead positions and no sign of complications.

The postoperative 12-lead ECG showed mostly AV sequential pacing, with occasional spontaneous $\mathrm{P}$ waves followed by native QRS (Fig. 1). The third complex of the tracing shows a shortened paced AV delay of $110 \mathrm{~ms}$. Is this normal pacemaker function?

\begin{abstract}
Answer
You will find the answer elsewhere in this issue.

Conflict of interest None of the authors have any conflict of interest related to this report.

Open Access This article is distributed under the terms of the Creative Commons Attribution License which permits any use, distribution, and reproduction in any medium, provided the original author(s) and the source are credited.
\end{abstract}

A. Pinter, MD $(\bowtie) \cdot$ P. Dorian

St. Michael's Hospital, University of Toronto,

30 Bond Street,

Toronto, ON, M5B 1W8 Canada

e-mail: pintera@smh.ca

P. Dorian

e-mail: dorianp@smh.ca 
Fig. 1 Postoperative standard 12-lead ECG (1 mV/10 mm, $25 \mathrm{~mm} / \mathrm{s}$ paper speed)

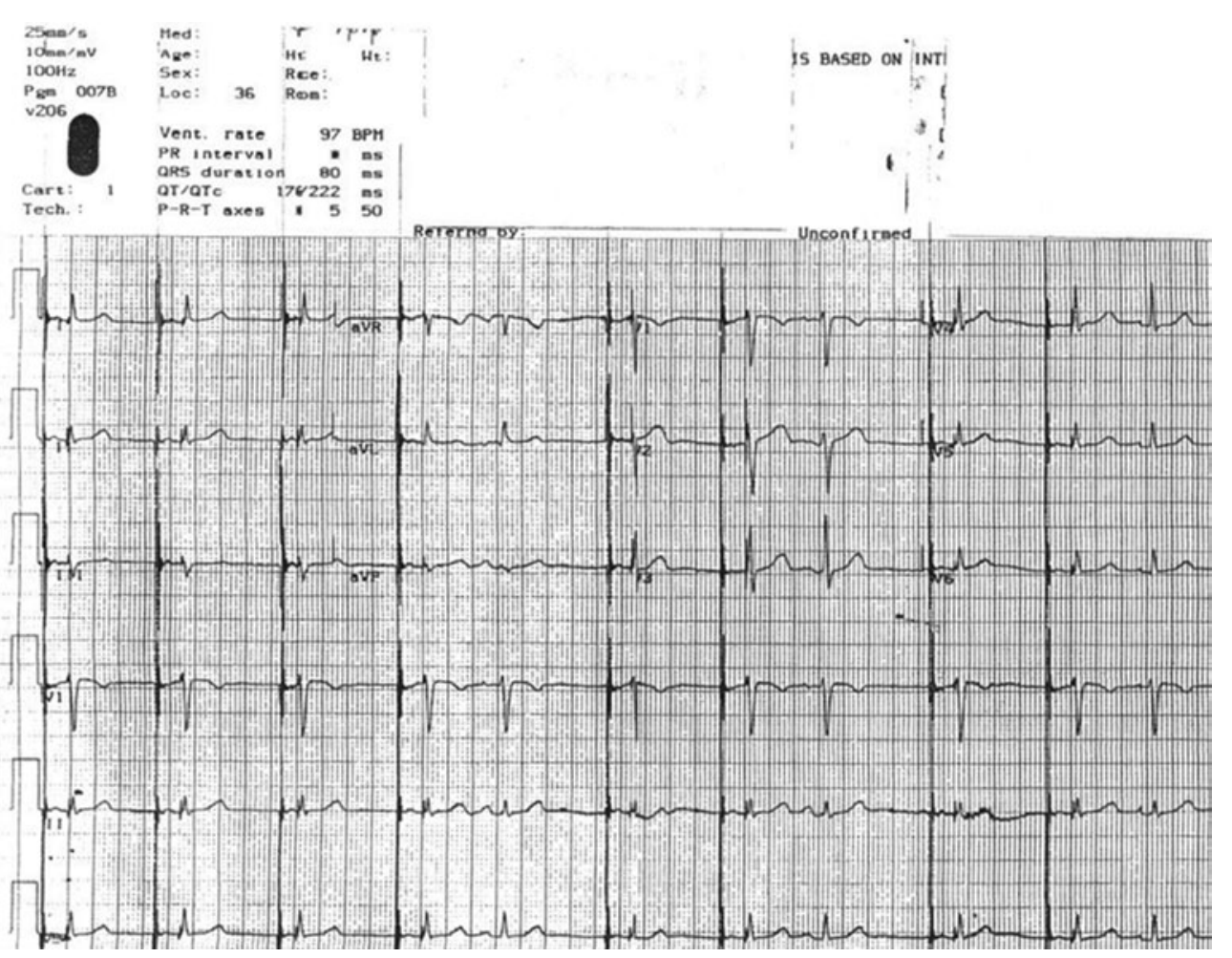

